\title{
Proximal Tibia Medial Open Wedge Osteotomy Using Puddu Plate for Treatment of Genu Varum in Adolescent and Young Patients
}

\author{
Waleed Faisal, Mohammed Elattar, Emad Abd-Elhadi \\ Orthopedic Surgery Dept. Zagazig University, Egypt \\ yousufmmkh@gmail.com
}

\begin{abstract}
Purpose: Medial open wedge high tibial osteotomy (MOWHTO) is an effective surgical procedure for patients who have medial compartmental osteoarthritis of the knee with varus deformity of the limb. The abnormal load on the medial compartment of the knee is directed to the lateral compartment with this procedure. A gap occurs on the proximal tibia while providing adequate correction. Filling this gap with bone grafts or synthetic materials has gained wide acceptance for preventing bone union problems or osteotomy site collapse. The aim of this study is to report our results of MOWHTOs performed without any bone graft or any other synthetic materials.

Patients and Methods: We evaluated 20patients MOWHTOs that have been performed between 2014 and 2018. Age of the patients ranged from 18 to 40. Fifteenpatients were females and five of them were males. The follow-up time was 12 months.

Results: All osteotomies united without loss of correction. The meanbone union time was 12.8 weeks. We did not have any major complication regarding the technique.

Conclusion: The results of our study have shown that we can achieve satisfactory and good results by performing MOWHTO procedure without using any bone grafts or synthetic materials.
\end{abstract}

Keywords: High tibial osteotomy, medial, open- wedge,puddu plate

\section{INTRODUCTION}

High tibial osteotomy is a procedure for young and active patients who have medial unicompartmental osteoarthritis of the knee and varus malalignment of the limb [1]. The aim of this procedure is to relocate the abnormal weight- bearing load from the medial arthritic compartment to the healthy lateral compartment. Medial opening-wedge technique is one of the high tibial osteotomy procedures [2]. There are numerous fixation techniques, bone grafts or augmentation materials for filling the gap on the osteotomy site [3-7]. Stable fixation is necessary for healing of the osteotomy and reducing the risk of non-union and loss of correction [8]. Stainless steel plates with conventional screws are first-generation plating systems. Current systems that provide more mechanical stability are locking titanium plates and locking screw designs [9-12]. It has been theoretically thought that bone union problems or correction loss due to osteotomy defect are the disadvantages of medial opening-wedge high tibial osteotomy (MOWHTO) [13]. Filling the osteotomy defect with bone grafts or any other materials for preventing these theoretical disadvantages has gained wide acceptance. There are various materials for filling the osteotomy defect (autograft, allograft, xenograft, bone substitute, acrylic cement and ceramic spacer) [14, 15]. Autologous bone graft has been accepted as 'gold standard' for this purpose [16]. 
Proximal Tibia Medial Open Wedge Osteotomy Using Puddu Plate for Treatment of Genu Varum in Adolescent and Young Patients

The aim of this study was to report our results of MOWHTO performed without any bone graft or any other synthetic materials.

\section{Patients ANd Methods}

Our study includes 20 MOWHTOs of 38 patients that have been performed between June 2014 and February 2018. The same surgeon performed all osteotomies. There were 15 females and five males aged ranging from 18 to 40. The inclusion criteria was patients to whom MOWHTO was performed for genu varum without any bone grafts and any synthetic materials. The indication for surgery was genu varum deformity and pain localized on medial knee compartment of the knee. The preoperative exclusion criteria were symptomatic osteoarthritis of the lateral or patellofemoral compartments, severe osteoarthritis, varus deformity of more than 15 , range of motion less than 90 and flexion contracture of more than 10. We evaluated clinical and radiologic process of each patient. Follow-up clinical and radiologic examinations were performed at the third week, sixth week, third month, sixth month, and 1 year.

\section{Surgical Procedure}

The patient was placed in a supine position on the operative table. The C-arm of an image intensifier was set up on the same side of the knee and opposite to the surgeon. The osteotomy procedure is performed through a vertical anteromedial incision extending 7 to $8 \mathrm{~cm}$ distally and parallel to the tibial axis (Fig. 1B).
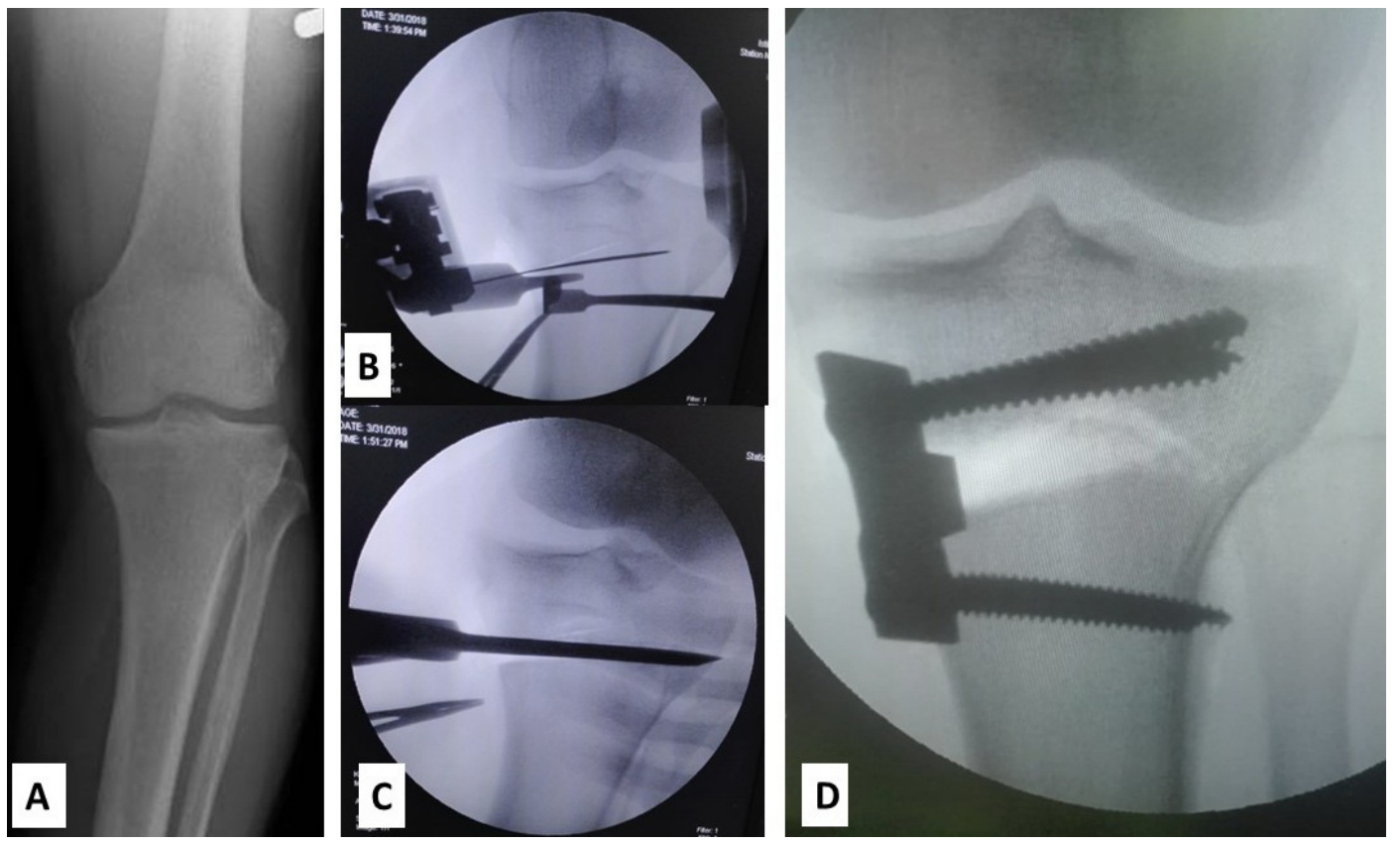

Figure1. case no-5:Male patient with g varum: A) Preoperative x-ray, B) Intraoperative photo during guide wire insertion, $\boldsymbol{C}$ ) Intraoperative photo during osteotomy, D) after plate fixation.

Sharp dissection is carried out beneath the skin incision to the pes anserinus and superficial medial collateral ligament (MCL), and detached from the tibia using Cobb elevator. Then, both pes anserinus and superficial MCL are retracted posteriorly and the posterior surface of the tibia at the level of the osteotomy is exposed. A retractor is placed dorsally in the osteotomy line (Fig. 3). This procedure provides complete exposure of the anteromedial surface of the tibia. The anterosuperior attachment site of the patellar tendon is exposed and a radiolucent retractor is placed under the patellar tendon. 
Proximal Tibia Medial Open Wedge Osteotomy Using Puddu Plate for Treatment of Genu Varum in Adolescent and Young Patients

\section{Osteotomy}

Under fluoroscopic control, a guide pin is drilled from the medial attachment site of the patellar tendon to the point $1.5 \mathrm{~cm}$ below the lateral joint line, and $1 \mathrm{~cm}$ medial to the lateral cortex. This guide pin is roughly directed toward the fibular head. Another guide pin is drilled parallel to the first pin and tibial posterior slope.

A cutting plate is placed through 2 guide pins, and the osteotomy is started $1 \mathrm{~mm}$ above the attachment site of the patellar tendon. A radiolucent retractor is used to protect the patellar tendon. A bone saw is used to cut 2 to $3 \mathrm{~cm}$ of the medial site of the tibia. Then, osteotomes are used to cut the tibia $1 \mathrm{~cm}$ medial to the lateral cortex (Figs. 1C). If the osteotomy is completed, it will easily open the osteotomized site. If not, anterior and/or posterior cortex is not completely cut. Next, the opener is inserted and gradually opened until desired correction. A Puddu plate (Arthrex Inc., Naple, FL) is placed into the osteotomy site (Figs. 1D). Plate positioning is important; if a plate is placed anteriorly, the tibial posterior slope will be increased.5

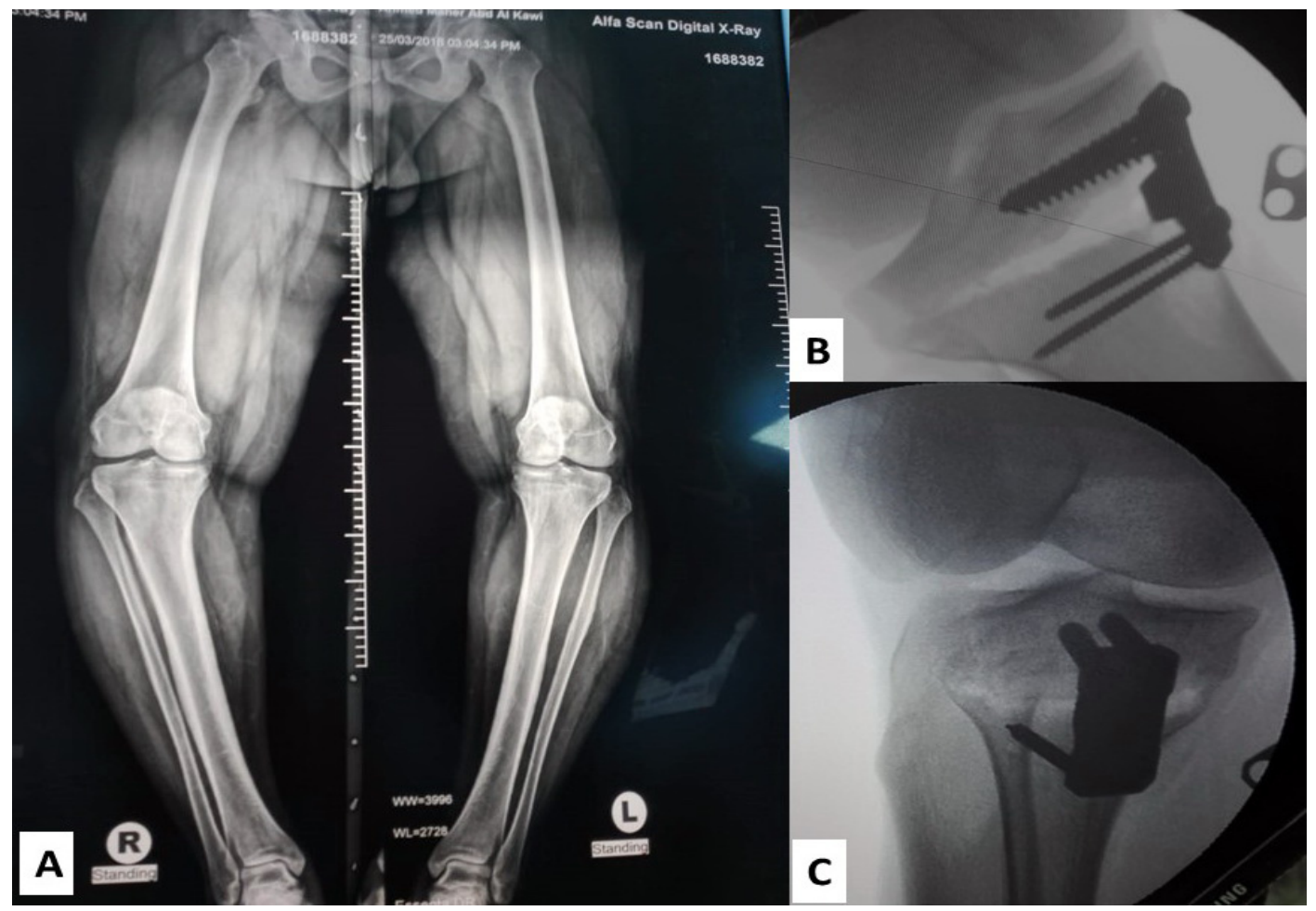

Figure2. pre and intraoperative planning for osteotomy: $\boldsymbol{A}$ ) preoperative scanogram, $\boldsymbol{B} \& \boldsymbol{C}$ ) intraoperative radiology

\section{Postoperative Period}

Early postoperative AP and lateral X-rays were taken (Fig. 3). Isometric quadriceps and active ankle exercises were started on the same day. Straight leg raising was started on the first day after surgery. For the next 3 weeks, patients were only allowed to move their knee from 0 to 30 of flexion in the hinge brace without weightbearing: After the first control at the end of the third week, patients were allowed to flex their knees up to 90 in the brace and also partial weight-bearing with crutches or walker was allowed as tolerated. Brace was removed and full flexion was stimulated after the second control that has been per-formed at the end of the sixth week, and also, radiologic evaluation was performed. Full weight-bearing was allowed after radiographic evaluation at the second control. Another patient's radiographs demonstrating the full union of the bone without loss of correction at the sixth month (Figs. 3). 
Proximal Tibia Medial Open Wedge Osteotomy Using Puddu Plate for Treatment of Genu Varum in Adolescent and Young Patients

\section{RESULTS}

All osteotomies united without early loss of correction at the end of the sixth month (Fig. 3). Mean time for bone union was 16.8 weeks (14-24). There was not any major complication. All of our patients were able to stand and walk on their operated legs 6 months after the procedure.

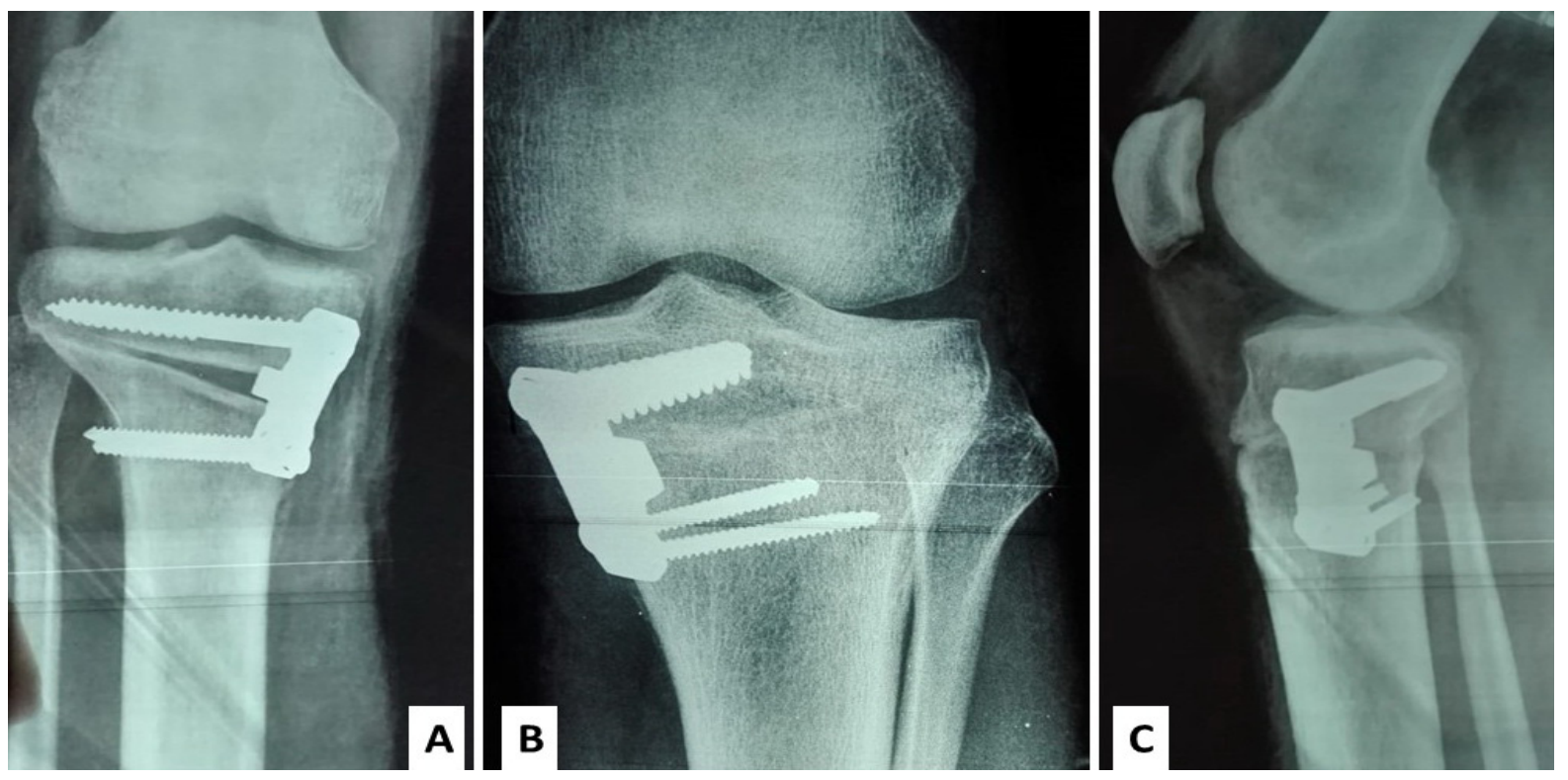

Figure3. plain $x$-ray postoperative and 1 year follow up.

\section{DISCUSSION}

There are many benefits of MOWHTO procedure when compared with lateral closing-wedge osteotomy. These benefits include easier procedure, preventing bone structure at the proximal tibia, obtaining more predictable correction, correcting the deformity close to its center and avoiding peroneal nerve, proximal tibiofibular joint and the anterior compartment injuries $[18,19]$. According to a report, despite its benefits, longer union time and requirement of bone grafts are handicaps of MOWHTO [20]. On the other hand, some authors reported that MOWHTO procedures had a higher risk of non-union when compared with closing-wedge osteotomies [21]. According to Gomoll [22] loss of correction and non-union are the two complications of the procedure, and according to him, non-union was related to the gap on the osteotomy site.

We think that the concerns about non-union and loss of correction forced the surgeons to use bone grafts and synthetic materials in many of the studies. However, the negative effects of grafts and synthetic materials are another cause of concern. Both of these concerns might decrease the surgeons' preference of MOWHTO procedure. Perhaps this is one of the reasons that MOWHTO procedure has not gain so much popularity as prosthetic replacements. We performed MOWHTOs without anygrafts or synthetic materials for protecting from the morbidity and negative effects of grafts and synthetic materials. Moreover, we did not encounter any non-union or any early loss of correction in our cases at 12-month follow-up.

Performing the osteotomy with no graft is supported by some authors [7]. Staubli et al. [8] reported their results of healing of MOWHTO without bone graft. They used a long plate for the procedure. However, there are some disadvantages of these long plates such as needing a longer or double incision for placing the plate and screws and needing a second surgery for removing them due to their large size. We used a short plate which can be applied easily. At the same time, removal of the plate is not essential due to its small size. 
Proximal Tibia Medial Open Wedge Osteotomy Using Puddu Plate for Treatment of Genu Varum in Adolescent and Young Patients

Zorzi et al. [2] have compared grafted and non-grafted osteotomies in their prospective randomized clinical trial. There were no significant differences about bone union between the two groups. However, they used spacers with plates. The difference of our study is that we did not use any spacers. The size of the healthy bone surface area is an important factor for bone healing. According to this classical knowledge, we support that any material like spacers that placed into the osteotomy site decreases the size of healthy bone surface area and so the union.

The finding of our study is that MOWHTO can be performed without any bone grafts or any other synthetic materials in fixation with locking titanium plates with locking screws without any spacer. Clinical and radiographic improvement occurred within 6 to 12 month after the surgery. There was not any non-union, and also, we did not see any early loss of correction.

A variety of grafts such as autografts or allografts and many kinds of synthetic materials have been largely used for avoiding non-union and loss of correction problems in MOWHTO procedure in the literature [23, 24].

The reason of using the bone grafts for filling the osteotomy gaps is to increase the mechanical stability and bone union. However, we think that keeping the lateral cortex intact and providing an intact lateral hinge together with applying locking plates and screws are sufficient for mechanical stability and bony union. Many authors considered that iliac bone autografts is the gold standard for this purpose [16]. However, donor-site morbidity is an important complication. Chronic pain, infections and paresthesia are possible donor-site morbidities of autologous iliac crest bone harvesting. Pollock et al [25] represented donor-site morbidity following iliac crest bone harvesting and its effect on walking. There are also other complications such as gluteal artery injury, deep wound infection, sciatic nerve injury related to this process [16, 26, and 27]. A review of 182 openingwedge high tibial osteotomy (OWHTO) demonstrated that the most common problem of OWHTO was bone graft harvest morbidity [16]. Chae et al. [28] reported three patients who have a linear fracture in the iliac bone related to autograft harvesting from the iliac wing. Discomfort in wearing clothes is another disadvantage tag of this procedure [29]. Finally, other unfavorable effects of the procedure are prolonged operation time, increased blood loss and palpable defects on the iliac crest [6]. Some authors advocate that decreasing blood loss from the osteotomy site, increasing the mechanical stability and bone healing are the advantages of using bone graft. We think that some bleeding from the osteotomized bone is necessary for the formation of hematoma that is essential for bone union. For this reason, we intentionally did not used drain for any case. Furthermore, harvesting an auto- graft from the iliac wing is a cause of increased blood loss itself. Additionally, the absence of any non-union or early loss of correction in our study has shown that filling the osteotomy site for increasing mechanical stability and bone healing might be reviewed again. Another option is to use allografts for MOWHTO procedure. There are some disadvantages of using allografts for MOWHTO procedure. These disadvantages are disease transmission, immunologic reactions and slow remodeling [23]. An alternative option for filling the osteotomy gap is to use of synthetic bone substitutes. However, there are also several disadvantagesof the technique such as delayed incorporation into bone, infections and soft tissue irritation [11,30]. Table 1 shows the complication rates of different techniques of MOWHTO [28]. Our reason for performing MOWHTO without bone grafts or any other synthetic materials depends on avoiding the complications of grafts and other synthetic materials. We believe that any of grafts, synthetic materials or spacers for plates are not essential for bone healing in but with locking plate fixationMOWHTO procedure. Considering mechanical and bio- logical reasons properly is the most important factor for bone healing and for successful outcomes.

\section{CONCLUSION}

Satisfactory and good results can beachieved by performing MOWHTO procedure without any kind of bone grafts or any synthetic augmentation materials so that we can avoid unnecessary morbidity and the risks of these materials.

American Research Journal of Orthopedics and Traumatology Page 5 
Proximal Tibia Medial Open Wedge Osteotomy Using Puddu Plate for Treatment of Genu Varum in Adolescent and Young Patients

\section{REFERENCES}

1. Franco V, Cerullo G, Cipolla M, Gianni E, Puddu G (2005) Osteotomy for osteoarthritis of the knee. Curr Orthop 19:415-427

2. Zorzi AR, da Silva HG, Muszkat C, Marques LC, Cliquet A Jr, de Miranda JB (2011) Opening-wedge high tibial osteotomy with and without bone graft. Artif Organs 35(3):301-307

3. Klinger HM, Lorenz F, Härer T (2001) Open wedge tibial oste- otomy by hemicallotasis for medial compartment osteoarthritis. Arch Orthop Trauma Surg 121(5):245-247

4. Koshino T, Murase T, Saito T (2003) Medial opening-wedge high tibial osteotomy with use of porous hydroxyapatite to treat medial compartment osteoarthritis of the knee. J Bone Joint Surg Am 85-A (1):78-85

5. Kraal T, Mullender M, de Bruine JH, Reinhard R, de Gast A, Kuik DJ, van Royen BJ (2007) Resorbability of rigid beta-tri- calcium phosphate wedges in open-wedge high tibial osteotomy: a retrospective radiological study. Knee 15(3):201-205

6. Sgaglione NA, Moynihan DP, Uggen C (2007). The use of allo- grafts in high tibial osteotomy: opening wedge technique. Oper Tech Sports Med 15:72-80

7. Stoffel K, Stachowiak G, Kuster M (2004) Open wedge high tibial osteotomy: biomechanical investigation of the modified Arthrex Osteotomy Plate (Puddu Plate) and the TomoFix Plate. Clin Biomech 19(9):944-950

8. Staubli AE, De Simoni C, Babst R, Lobenhoffer P (2003) To- moFix: a new LCP-concept for open wedge osteotomy of the medial proximal tibia-early results in 92 cases. Injury 34(Suppl 2):B55-B62

9. Dorsey WO, Miller BS, Tadje JP, Bryant CR (2006). The stability of three commercially available implants used in medial opening wedge high tibial osteotomy. J Knee Surg 19(2):95-98

10. Niemeyer P, Koestler W, Kaehny C, Kreuz PC, Brooks CJ, Strohm PC, Helwig P, Suedkamp NP (2008) Two-year results of open-wedge high tibial osteotomy with fixation by medial plate fixator for medial compartment arthritis with varus malalignment of the knee. Arthroscopy 24(7):796-804

11. Spahn G (2004) Complications in high tibial (medial opening wedge) osteotomy. Arch Orthop Trauma Surg 124(10):649-653.

12. Spahn G, Mu"ckley T, Kahl E, Hofmann GO (2006) Biome- chanical investigation of different internal fixations in medial opening-wedge high tibial osteotomy. Clin Biomech 21(3):272-278

13. Amendola A, Panarella L (2005) High tibial osteotomy for the treatment of unicompartmental arthritis of the knee. Orthop Clin N Am 36(4):497-504

14. Brosset T, Pasquier G, Migaud H, Gougeon F (2011) Opening wedge high tibial osteotomy performed without filling the defect(TomoFix ${ }^{\mathrm{TM})}$ and early weight-bearing: prospective evaluation of bone union, precision and maintenance of correction in 51 cases. Orth tramatolsurg; Res 97(7):705-711

15. Levai JP, Bringer O, Descamps S, Boisgard S (2003) Xenograft- related complications after filling valgus open wedge tibial osteotomy defects. Rev Chir Orthop ReparatriceAppar Mot 89(8):707-711

16. Warden SJ, Morris HG, Crossley KM, Brukner PD, Bennell KL (2005) Delayed- and non-union following opening wedge high tibial osteotomy: surgeons' results from 182 completed cases. Knee Surg Sports Traumatol Arthrosc 13(1):34-37 
Proximal Tibia Medial Open Wedge Osteotomy Using Puddu Plate for Treatment of Genu Varum in Adolescent and Young Patients

17. Wade R, Richardson J (2001) Outcome in fracture healing: areview. Injury 32:109-114

18. Rose T, Imhoff AB (2007) Complications after transgenicular osteotomies. Oper Tech Orthop 17:80-86

19. Wright JM, Crockett HC, Slawski DP, Madsen MW, Windsor RE (2005) High tibial osteotomy. J Am Acad Orthop Surg13(4):279-289

20. Ganji R, Omidvar M, Izadfar A, Alavinia SM (2013) Opening wedge high tibial osteotomy using tibial wedge allograft: a case series study. Eur J Orthop Surg Traumatol 23(1):111-114

21. Marti RK, Verhagen RA, Kerkhoffs GM, Moojen TM (2001) Proximal tibial varus osteotomy. Indications, technique, and five to twenty-one-year results. J Bone Joint Surg Am 83-A (2): 164-170

22. Gomoll AH (2011) High tibial osteotomy for the treatment of unicompartmental knee osteoarthritis: a review of the literature, indications, and technique. Phys Sportsmed 39(3):45-54

23. Aryee $\mathrm{S}$, Imhoff $\mathrm{AB}$, Rose $\mathrm{T}$, Tischer $\mathrm{T}$ (2008) Do we need synthetic osteotomy augmentation materials for opening-wedge high tibial osteotomy. Biomaterials 29(26):3497-3502

24. Kuremsky MA, Schaller TM, Hall CC, Roehr BA, Masonis JL (2010) Comparison of autograft versus allograft in opening- wedge high tibial osteotomy. J Arthroplast 25(6):951-957

25. Pollock R, Alcelik I, Bhatia C, Chuter G, Lingutla K, Budithi C, Krishna M (2008) Donor site morbidity following iliac crest bone harvesting for cervical fusion: a comparison between minimally invasive and open techniques. Eur Spine J 17(6):845-852

26. Amendola A, Bonasia DE (2010) Results of high tibial osteot- omy: review of the literature. Int Orthop 34(2):155-160

27. Brull SJ, Lieponis JV, Murphy MJ, Garcia R, Silverman DG (1992) Acute and long-term benefits of iliac crest donor site perfusion with local anesthetics. AnesthAnalg 74(1):145-147

28. Chae DJ, Shetty GM, Wang KH, Montalban AS Jr, Kim JI, Nha KW (2011): Early complications of medial opening wedge high tibial osteotomy using autologous tricortical iliac bone graft and T-plate fixation. Knee 18(4):278-284

29. Ahlmann E, Patzakis M, Roidis N, Shepherd L, Holtom P (2002) Comparison of anterior and posterior iliac crest bone grafts in terms of harvest-site morbidity and functional outcomes. J Bone Joint Surg Am 84-A (5):716-720

30. van Hemert WL, Willems K, Anderson PG, van Heerwaarden RJ, Wymenga AB (2004) Tricalcium phosphate granules or rigid wedge preforms in open wedge high tibial osteotomy: a radio- logical study with a new evaluation system. Knee 11(6):451-456

Citation: Waleed Faisal, Mohammed Elattar, Emad Abd-Elhadi. "Proximal Tibia Medial Open Wedge Osteotomy Using Puddu Plate for Treatment of Genu Varum in Adolescent and Young Patients". American Research Journal of Orthopedics and Traumatology. 2018; 3(1): 1-7.

Copyright (c) 2018 Waleed Faisal, Mohammed Elattar, Emad Abd-Elhadi. This is an open access article distributed under the Creative Commons Attribution License, which permits unrestricted use, distribution, and reproduction in any medium, provided the original work is properly cited.

American Research Journal of Orthopedics and Traumatology

Page 7 